Vol. $12 \mathrm{~N}^{0} 2$, pp.129-135 Decenber 1993

Universidad Católica del Norte

Antofagasta - Chile

\title{
EVEN MORE ON THE FUNDAMENTAL THEOREM OF CALCULUS
}

\author{
CHARLES SWARTZ \\ New Mexico State University, New Mexico, USA.
}

\begin{abstract}
We show that the general form of the Fundamental Theorem of Calculus for Lipschitz functions derived by Botsko [B2] is valid for the gauge integral and can be obtained by very elementary means.
\end{abstract}


In [B2] Botsko points our that the usual form of the Fundamental Theoren of Calculus (FTC) for the Riemann integral which appears in most standard real analysis books does not apply to such a simple function as $f(x)=0$ for $-1 \leq x<0$ and $f(x)=1$ for $0 \leq x \leq 1$. Botsko proceeds to give an elementary proof of a more general form of the FTC which does apply to such functions as $f$. As a corollary of this general form of the FTC, he derives the following result.

Theorem 1. Let $f:[a, b] \longrightarrow \mathbb{R}$ be Riemann integrable and let $g$ be a continuous function sucl that $g^{\prime}=f$ except on a countable set. Then $\int_{a}^{b} f(x) d x=g(b)-b(a)$.

Note that Theorem 1 is applicable to the function $f$ above (take $g(x)=0$ for $-1 \leq x<0$ and $g(x)=x$ for $0 \leq x \leq 1)$. Note also that the continuity of $g$ is important (take $g_{1}(x)=0$ for $-1 \leq x<0$ and $g_{1}(x)=x+1$ for $0 \leq x \leq 1$ ).

The important (annoying?) hypothesis in Theorem 1 is the need to assume that the function $f$ is integrable. In the note [ST], it was pointed out that for functions which are differentiable everywhere the FTC holds in full generality if instead of the Riemann (or Lebesgue) integral, the gauge integral is used. Moreover, it was shown in [ST] that Theorem 1 is valid for the gauge integral without the assumption that $f$ is integrable, i.e, such functions are automatically gauge integrable.

We would like to point out that the general form of the FTC for Lipschitz functions derived by Botsko is valid for the gauge integral and can be obtained by very elementary means.

We begin by giving the definition of the gauge integral. Any positive function $\delta:[a, b] \longrightarrow \mathbb{R}$ is called a gauge. A tagged division of an interval $[a, b]$ is a finite set

$$
T=\left\{x_{0}, x_{1}, \ldots, x_{n} ; t_{0}, t_{1}, \ldots, t_{n-1}\right\}
$$

such that $\left\{x_{0}, x_{1}, \ldots, x_{n}\right\}$ is a partition of $[a, b]$ and $t_{i} \in\left[x_{i}, x_{i+1}\right]$; the point $t_{i}$ is called a tag for the subinterval $\left[x_{i}, x_{i+1}\right]$. If $\delta$ is a hauge, a tagged division is said to be $\delta$-finite if

$$
\left[x_{i}, x_{i+1}\right] \subset\left(t_{i}-\delta\left(t_{i}\right), t_{i}+\delta\left(t_{i}\right)\right)
$$

Definition 2. A function $f:[a, b] \longrightarrow \mathbb{R}$ is gauge integrable over $[a, b]$ if there exists $A \in \mathbb{R}$ such that for every $\varepsilon>0$ there is a gauge $\delta$ on $[a, b]$ such that if $T$ is a $\delta$-fine tagged division of $[a, b]$, then

$$
\left|\sum_{i=0}^{n-1} f\left(t_{i}\right)\left(x_{i+1}-x_{i}\right)-A\right|<\varepsilon .
$$


The number $A$ is called the gauge integral of $f$ and is denoted by $\int_{a}^{b} f$; all subsequent integrability statements will refer to the gauge integral. The gauge integral is also referred to as the generalized Riemann integral [Mc] or the Riemann complete integral [H]. Note that a function is Riemann integrable if and only if it is gauge integrable with the gauge a positive, constant function. It is the ability to vary the gauge which makes the gauge integral so powerful; this is illustrated in the proof of Lemma 3 below.

Although the gauge integral is obtained from only a slight variation of the Riemann integral, it was discovered only in 1956 by J. Kurzweil $([\mathrm{K}])$. It was rediscovered independently by $\mathrm{R}$. Henstock $([\mathrm{H}])$ who developed the major convergence theorems for the gauge integral.

For Botsko's version of the FCT we need the notion of a set of (Lebesgue) measure 0 . A subset $Z \subset \mathbb{R}$ has measure 0 if for every $\varepsilon>0$ there is a countable sequence of open intervals $\left\{\left(a_{i}, b_{i}\right)\right\}$ such that $Z \subset \cup_{i}\left(a_{i}, b_{i}\right)$ and $\sum_{i}\left(b_{i}-a_{i}\right)<\varepsilon$. A property is said to hold almost everywhere (a.e.) if it holds except on a set of measure 0 .

In our derivation of the $\mathrm{F} T \mathrm{C}$ for the gauge integral, the following result is useful.

Lemma 3. If $f:[a, b] \longrightarrow \mathbb{R}$ is 0 a.e. in $[a, b]$, then $f$ is integrable and $\int_{a}^{b} f=0$.

Proof. Let $Z$ be the set where $f$ is non-zero and set $Z_{i}=\{t \in Z: i-1<|f(t)| \leq$ $i$ ) for $i \in \mathbb{N}$. Let $\varepsilon>0$. For each $i$ there is an open set $G_{i} \supset Z_{i}$ such that $G_{i}$ is a countable union of open intervals the suin of whose lengths is less than $\varepsilon / i 2^{i}$. Define a gauge on $[a, b]$ as follows: if $t \notin Z$, set $\delta(t)=1$, and if $t \in Z_{i}$, pick $\delta(t)>0$ such that $(t-\delta(t), t+\delta(t)) \subset G_{i}$. Now suppose that

$$
T=\left\{x_{0}, x_{1}, \ldots, x_{n} ; t_{0}, t_{1}, \ldots, t_{n-1}\right\}
$$

is a $\delta$-fine tagged partition of $[\boldsymbol{a} . \boldsymbol{b}]$. Consider the Riemann sum

$$
\left|\sum_{k=0}^{n-1} f\left(t_{k}\right)\left(x_{k+1}-x_{k}\right)\right|
$$

If $t_{k} \notin Z, f\left(t_{k}\right)=0$, while if $t_{k} \in Z$, then $t_{k}$ belongs to exactly one of the $Z_{i}$ so $\left|f\left(t_{k}\right)\left(x_{k+1}-x_{k}\right)\right| \leq i\left|x_{k+1}-x_{k}\right|<i \varepsilon / i 2^{i}=\varepsilon / 2^{i}$. Hence, the sum in (1) is less than $\varepsilon$ and the lemma is established.

Note how the gauge $\delta$ in the proof of Lemma 3 is chosen to vary with the point $t$. 
The gauge integral is linear so if $g$ is gauge integrable and $f=g$ a.e., then $f$ is gauge integrable and $\int_{a}^{b} g=\int_{a}^{b} f$. This observation is used in the proof of Theorem 5 below.

In proving versions of the FTC for the gauge integral the following lemma is useful.

Lemma 4. (Straddle Lemma). Let $g:[a, b] \longrightarrow \mathbb{R}$ be differentiable at $z \in[a, b]$. Then for each $\varepsilon>0$, there is a $\delta>0$ such that $\left|g(v)-g(u)-g^{\prime}(z)(v-u)\right| \leq \varepsilon(v-u)$, whenever $u \leq z \leq v$ and $[u, v] \subset[a, b] \cap(z-\delta, z+\delta)$.

The geometric meaning of the Straddle Lemma is clear, when $u$ and $v$ "straddle" $z$, the slope of the chord between the points $(u, f(u))$ and $(v, f(v))$ is close to the slope of the tangent line at $(z, f(z))$. For a proof of the Straddle Lemma, see [DS], [Mc] or [ST].

We now give a version of Botsko's FTC for the gauge integral. Note that weaker differentiation assumptions on the function $g$ requires stronger continuity assumptions.

Theorem 5. Let $g:[a, b] \longrightarrow \mathbb{R}$ satisfy a Lipschitz condition and $f:[a, b] \longrightarrow \mathbb{R}$ be such that $g^{\prime}=f$ a.e.

Then $f$ is integrable and $\int_{a}^{b} f=g(b)-g(a)$.

Proof. Assume $|g(s)-g(t)| \leq L|s-t|$ for $s, t \in[a, b]$.

By Lemma 3 we may assume that $f(t)=0$ when $g$ is not differentiable at $t$. Let $Z$ be the set of measure 0 where $g^{\prime}$ fails to exit. Let $\varepsilon>0$. Let $G$ be an open set containing $Z$ which is a countable union of open intervals the sum of whose lengths is less than $\varepsilon$. Define a gauge $\delta$ as follows: if $t \in Z$, pick $\delta(t)>0$ as in the Straddle Lemma and if $t \in Z$, pick $\delta(t)>0$ such that $(t-\delta(t), t+\delta(t)) \subset G$. Let $\left\{x_{0}<x_{1}<\ldots<x_{n} ; t_{0}, t_{1}, \ldots, t_{n-1}\right\}$ be a $\delta$-fine tagged partition of $[\mathrm{a}, \mathrm{b}]$ Consider the sum

$$
\left|\sum_{i=0}^{n-1} f\left(t_{i}\right)\left(x_{i+1}-x_{i}\right)-(g(b)-g(a))\right|=\left|\sum_{i=1}^{n-1} f\left(t_{i}\right)\left(x_{i+1}-x_{i}\right)-\left(g\left(x_{i+1}\right)-g\left(x_{i}\right)\right)\right| .
$$

We break the sum on the right hand side into parts; let $\sum^{\prime}$ denote the sum of the terms with tags $t_{i} \in Z$ and $\sum^{\prime \prime}$ the sum of the terms with tags $t_{i} \in Z$. If $t_{i} \in Z$, then by the Straddle Lemma

$$
\left|f\left(t_{i}\right)\left(x_{i+1}-x_{i}\right)-\left(g\left(x_{i+1}\right)-g\left(x_{i}\right)\right)\right|<\varepsilon\left|x_{i+1}-x_{i}\right|
$$


so $\sum^{\prime}<\varepsilon(b-a)$. If $t_{i} \in Z$, then $f\left(t_{i}\right)=0$ and $\left|g\left(x_{i+1}\right)-g\left(x_{1}\right)\right| \leq L\left|x_{i+1}-x_{i}\right|$; since $\left[x_{i}, x_{i+1}\right] \subset G$ for each $i, \Sigma^{\prime \prime} \leq L \varepsilon$. Hence, the sum on the right hand side of $(2)$ is less than $\varepsilon(b-a+L)$, and it follows that $f$ is gauge integrable, and its integral is $g(b)-g(a)$.

In Botsko's version of Theoren 5 for the Riemann integral, be must assume the integrability of the function $f$; the function $f$ is automatically gauge integrable.

As an application of Theoren 5, we have

Corollary 6. If $g$ satisfies a Lipschitz condition and $g^{\prime}=0$ a.e., then $g=$ constant.

Botsko lıas given an elementary proof of this result in [B1]; he used it in [B1] to show that a function which is bounded and continuous a.e. in Riemann integrable. He also used it in [B2] in his derivation of the FTC for Lipsclitz functions.

The proof of Theorem 5 can be easily modified to apply to absolutely continuous functions. Recall that a function $g:[a, b] \longrightarrow \mathbb{R}$ is absolutely continuous if for every $\varepsilon>0$ there exists $\eta>0$ such that whenever $\left[\left(a_{i}, b_{i}\right)\right]_{i=1}^{n}$ is a finite sequence of disjoint open intervals satisfying $\sum_{i=1}^{n}\left(b_{i}-a_{i}\right)<\eta$, then $\sum_{i=1}^{n}\left|g\left(b_{i}\right)-g\left(a_{i}\right)\right|<\varepsilon$. Lipschitz functions are obviously absolutely continuous; $g(t)=\sqrt{t}, 0 \leq t \leq 1$, is an absolutely continuous function which is not Lipschitz. For absolutely continuous functions, we have the following form of the FTC.

Theorem 7. Let $g:[a, b] \longrightarrow \mathbb{R}$ be absolutely continuous and $f:[a, b] \longrightarrow \mathbb{R}$ be such that $g^{\prime}=f$ a.e.

Then $f$ is integrable and $\int_{a}^{b} f=g(b)-g(a)$.

Proof. As in Theorem 5 let $Z$ be the set where $g^{\prime}$ does not exists. Let $\varepsilon>0$ and let $\eta>0$ be as in the definition of absolute continuity of $g$. Let $G$ be an open set containing $Z$ which is a countable union of open intervals the sum of whose lengths is less than $\eta$. Define a gauge $\delta$ as in Theorem 5 . If we proceed as in the proof of Theorem 5 , when $t_{i} \in Z, f\left(t_{i}\right)=0$, and $\left[x_{i}, x_{i+1}\right] \subset G$ so the sum of the lengths of these intervals in less than $\eta$, and $\sum^{\prime \prime}<\varepsilon$ by the absolute continuity of $g$. Thus, the sum on the right hand side of $(2)$ is less than $\varepsilon(b-a+1)$ and the result follows as before.

As before, we have an immediate corollary of Theorem 7.

Corollary 8. If $g:[a, b] \longrightarrow \mathbb{R}$ is absolutely continuous and $g^{\prime}=0$ a.e., then $g=$ constant. 

18.15).

This result in often proved by using the Vitali Covering Theorem ([HS]

Theorem 7 loolds for the Lebesgue integral and is sonetimes called the FTC for the Lebesgue integral ([HS] 18.16). It turus out that absolutely continuous (and, lience, Lipschitz) functions are differentiable a.e. so the differentiability hypothesis in Theorems 5 and 7 is superfluous; however, the proofs of this fact usually depend on covering arguments and are not considered to be elementary (see [IS]).

Although the gauge integral has a very elementary definition, it is a very powerful integral and is actually more general than the Lebesgue integral. For example, the monotone and dominated convergence theorems hold for the gauge integral and all improper Riemann integrable functions are gauge integrable. Because of its elementary nature, the gauge integral would appear to be a natural candidate for inclusion in introductory real analysis courses. Elementary expositions of the gauge integral in English can be found in [DS], [L] and [Mc].

\section{REFERENCES}

[BI] Botsko, M.: All elementary proof that a bounded a.e. continuous function is Riemann integrable. Amer. Math. Monthly, 95 (1988), 249-252.

[B2] Botsko, M.: A Fundamental Theorem of Calculus that applies to all Riemann integrable functions. Math. Mag., 64 (1991), 347-348.

[DS] DePree, J.; Swartz, C.: Introduction to Real Analysis. Wiley, N.Y., 1987.

[H] Henstock, R.: Definitions of Riemann type of the variational integrals. Proc. London Math. Soc., 11(1961), 402-418.

[HS] Hewitt, E.; Stromberg, K.: Real and Abstract Analysis. Springer-Verlag, Heidelberg, 1965.

[K] Kurzweil, J.: Generalized ordinary differential equations and continuous dependence on a parameter. Czech. Math. J., 82 (1957), 418-449.

[L] Lee Peng Yee: Lanzhou Lectures on Henstock Integration. World Scientific Publs., Singapore, 1989.

[Mc] McLeod, R.: The Generalized Riemann Integral. Carus Math. Monograph 20, $M A A, 1980$.

[ST] Swartz, C.; Thomson, B.: More on the Fundamental Theorem of Calculus. Amer. Math. Monthly, 95 (1988), 644-648. 
Received: January 15, 1993.

Charles Swartz

Departnent of Mathematical Sciences

New Mexico Stato University

Las Cruces, NM 88003, USA. 